\title{
Stability of High-Order Linear Itô Equations with Delays
}

\author{
Lev Idels ${ }^{*}$, Ramazan Kadiev², Arcady Ponosov³ \\ ${ }^{1}$ Department of Mathematics, Vancouver Island University, Nanaimo, Canada \\ ${ }^{2}$ Dagestan Research Center of the Russian Academy of Sciences \& Department of Mathematics, Dagestan \\ State University, Makhachkala, Russia \\ ${ }^{3}$ Faculty of Sciences and Technology, Norwegian University of Life Sciences, Ås, Norway \\ Email: ^lev.idels@viu.ca, kadiev_r@mail.ru, arkadi@nmbu.no
}

How to cite this paper: Idels, L., Kadiev, R. and Ponosov, A. (2018) Stability of High-Order Linear Itô Equations with Delays. Applied Mathematics, 9, 250-263. https://doi.org/10.4236/am.2018.93019

Received: February 7, 2018

Accepted: March 26, 2018

Published: March 29, 2018

Copyright $\odot 2018$ by authors and Scientific Research Publishing Inc. This work is licensed under the Creative Commons Attribution International License (CC BY 4.0).

http://creativecommons.org/licenses/by/4.0/

\begin{abstract}
A novel general stability analysis scheme based on a non-Lyapunov framework is explored. Several easy-to-check sufficient conditions for exponential $p$-stability are formulated in terms of $\mathcal{M}$-matrices. Stability analysis of applied second-order Itô equations with delay is provided as well. The linearization technique, in combination with the tests obtained in this paper, can be used for local stability analysis of a wide class of nonlinear stochastic differential equations.
\end{abstract}

\section{Keywords}

High-Order Stochastic Models, Delay, Stability, Non-Lyapunov Methods, $\mathcal{M}$-Matrices

\section{Introduction}

Stochastic differential equations (SDE) of the second and higher order with or without time-varying delays, naturally appear in multiple applications, where deterministic models are perturbed by the white noise or its generalizations. A classical example is the Langevin equation (see e.g. [1]). Liénard-type second-order stochastic equations were examined in multiple papers (see, for example, [2] [3] [4] and [5]). In [4] explicit conditions for asymptotic stability of the second-order equation under additive white noise were obtained. In [6] boundedness and exponential stability conditions for second-order SDEs with a constant delay were examined. Other examples deal with the study of sensibility on stochastic perturbations of regenerative vibrations in milling process described by the second order linear differential equation with delays [7]; a planar inverted pendulum on a cart, operating under modelling uncertainties 
and stochastic perturbations, modeled by the two-dimensional subsystem in [8].

Stochastic high-order models of processes related to abrasive waterjet milling or fluid energy milling (batch grinding) are well-known as well (see e.g. [9]). Stochastic high-order feedforward nonlinear system with time-varying delay was suggested to model many physical devices, such as the ball-beam with a friction term and the cart-pendulum system [10]. Large-scale stochastic high-order systems with time-varying delay are described by a series of interconnected subsystems in [3]. A hyperjerk system is a dynamical system governed by an $n$-th order ordinary differential equation with $n>3$ describing the time evolution of a single scalar variable (see e.g. [11]), which can also be perturbed by a stochastic noise.

Several definitions of stochastic Lyapunov stability are used in the literature, e.g. stability in probability, stability in the mean and almost sure stability, stability of the $p$-th mean ( $p$-stability), and even more. For applications to real systems, stability properties that are close to deterministic stability (almost sure sample stability) are the most desired, while conditions for $p$-stability are technically easier to obtain.

In this paper we study the global $p$-stability of the linear $n$-th order Itô delay equation

$$
\begin{aligned}
\mathrm{d} x^{(n-1)}(t)= & {\left[-\sum_{j=0}^{n-1} a_{j 0} x^{(j)}(t)+\sum_{j=1}^{m_{0}} c_{j 0} x\left(t-\tau_{j 0}\right)\right] \mathrm{d} t } \\
& +\sum_{l=1}^{m}\left[-\sum_{j=0}^{n-1} a_{j l}(t) x^{(j)}(t)+\sum_{j=1}^{m_{l}} c_{j l}(t) x\left(t-\tau_{j l}\right)\right] \mathrm{d} \mathcal{B}_{l}(t)(t \geq 0),
\end{aligned}
$$

and its non-autonomous generalization

$$
\begin{aligned}
\mathrm{d} x^{(n-1)}(t)= & {\left[-\sum_{j=0}^{n-1} a_{j 0}(t) x^{(j)}(t)+\sum_{j=1}^{m_{0}} c_{j 0}(t) x\left(h_{j 0}(t)\right)\right] \mathrm{d} t } \\
& +\sum_{l=1}^{m}\left[-\sum_{j=0}^{n-1} a_{j l}(t) x^{(j)}(t)+\sum_{j=1}^{m_{l}} c_{j l}(t) x\left(h_{j l}(t)\right)\right] \mathrm{d} \mathcal{B}_{l}(t)(t \geq 0),
\end{aligned}
$$

where $\mathcal{B}_{l}(t)$ are independent scalar Brownian motions defined on a probability space.

Stability of stochastic first-order differential equations with delays, as well as systems of equations, has been extensively studied (see [2] [4] [5] [6] [12] [13], [14] [15] [16] and the references therein). The main tool for studying the global stability is the Lyapunov functional method and its stochastic modifications (see e.g. [2] and the references therein). While this method has been and remains the leading technique, numerous difficulties with the theory and applications to specific problems, even in the deterministic case, persist. It is, in particular, the case if one examines stability of high-order differential equations. Of course, one can always apply the Lyapunov method after reducing a high-order equation to a first-order system in the well-known way, and this technique does work in specific situations. Yet, this approach may also fail in many cases. That is why, very few papers in recent literature have examined stability of high-order stochastic differential equations with time-varying delays as such. 
In the recent paper [17], a new, more flexible algorithm of reducing a high-order deterministic differential equation with delay to a first-order system was suggested. The approach goes back to the theory of $\mathcal{M}$-matrices. This idea is not new: absolute stability via $\mathcal{M}$-matrices was studied in the monograph [18], while in the more recent paper [19] this technique was applied to stability of neural networks. The efficiency of this method in connection with high-order deterministic equations was demonstrated in [17]. In this paper we claim that this approach is applicable to the SDE with delays as well, provided that the analysis based on $\mathcal{M}$-matrices is combined with the regularization method from [14]. The latter method differs from the classical Lyapunov technique, which presupposes the existence of suitable Lyapunov functionals. Rather, the method from [14] requires the existence of a suitable auxiliary equation which is used to regularize the original equation and subsequently to check solvability of a regularized equation in a carefully chosen space of stochastic processes.

In conclusion, we stress that even if this paper studies stochastic linear equations, the various linearization criteria for a nonlinear stochastic differential equation (see e.g. [20] and the references therein) in combination with the tests obtained in this paper, can be used to examine the local stability of a nonlinear stochastic differential equations.

\section{Preliminaries}

Let $\left(\Omega, \mathcal{F},(\mathcal{F})_{t \geq 0}, P\right)$ be a stochastic basis, where $\Omega$ is set of elementary probability events, $\mathcal{F}$ is a $\sigma$-algebra of all events on $\Omega,(\mathcal{F})_{t \geq 0}$ is a right continuous family of $\sigma$-subalgebras of $\mathcal{F}, P$ is a probability measure on $\mathcal{F}$; all the above $\sigma$-algebras are assumed to be complete w.r.t. $P$, i.e. containing all subsets of zero measure; the symbol $E$ stands below for the expectation related to the probability measure $P$. The expectation (the integral w.r.t. the measure $P$ ) is denoted by $E$.

We will use the following notations:

- $|$.$| is an arbitrary yet fixed norm in R^{n},\|$.$\| being the associated matrix$ norm.

- $\quad \mu$ is the Lebesgue measure on $[0,+\infty)$.

- $\quad\|\cdot\|_{X}$ is the norm in a normed space $X$.

- $\quad p$ is an arbitrary real number satisfying $1 \leq p<\infty$.

- $\left(\mathcal{B}_{1}, \cdots, \mathcal{B}_{m}\right)$ is the standard $m$-dimensional Brownian motion (i.e. the scalar Brownian motions $\mathcal{B}_{l}$ are all independent).

Recall that the classic Marcinkiewicz-Zygmund inequality

$$
\left(E\left|\sum X_{i}\right|^{2 p}\right)^{1 / 2 p} \leq \rho_{p}\left(E\left|\sum X_{i}^{2}\right|^{p}\right)^{1 / 2 p}
$$

where $X_{i}$ are independent random variables with the zero mean, can be extended to the integral form

$$
\left(E\left|\int_{0}^{t} f(s) \mathrm{d} \mathcal{B}_{l}(s)\right|^{2 p}\right)^{1 / 2 p} \leq \rho_{p}\left(E\left(\int_{0}^{t}|f(s)|^{2} \mathrm{~d} s\right)^{p}\right)^{1 / 2 p}
$$


for any predictable stochastic process $f(s)(0 \leq s \leq t)$, any $t>0$ and any component $\mathcal{B}_{l}(s) \quad(1 \leq l \leq m)$ of the Brownian motion $\mathcal{B}$. The inequality (4) is often used in this paper. In 1988 D.L. Burkholder proved (see for example [21], [22]) that in the Marcinkiewicz-Zygmund inequality (3) the constant $\rho_{p}=2 p-1$ is best possible for all for $p \geq 1$.

Equation (2) is assumed to be equipped with the initial conditions

$$
x(t)=\varphi(t)(t<0),
$$

and

$$
x^{(j)}(0)=b_{j+1}, \quad j=0, \cdots, n-1,
$$

where

1) $a_{j l}, l=0, \cdots, m, j=0, \cdots, n-1, \quad c_{j l}, l=0, \cdots, m, j=1, \cdots, m_{l}$ are Lebesgue measurable functions defined on $[0, \infty)$; in addition, we assume that $0<\hat{a}_{j 0} \leq a_{j 0}(t) \leq A_{j 0} \quad \mu$-almost everywhere for some positive constants $\hat{a}_{j 0}, A_{j 0}, j=0, \cdots, n-1,\left|a_{j l}(t)\right| \leq A_{j l} \quad \mu$-almost everywhere for some positive constants $A_{j l}, l=1, \cdots, m, j=0, \cdots, n-1$, and $\left|c_{j l}(t)\right| \leq c_{j l} \quad \mu$-almost everywhere for some positive constants $c_{j l}, l=0, \cdots, m, j=0, \cdots, m_{l}$.

2) $h_{j l}, l=0, \cdots, m, j=1, \cdots, m_{l}$ are Lebesgue measurable functions defined on $[0, \infty)$ and satisfying the estimates $0 \leq t-h_{j l}(t) \leq \tau_{j l} \quad \mu$-almost everywhere for some positive constants $\tau_{j l}, l=j, \cdots, m, j=1, \cdots, m_{l}$.

3) $\varphi$ is an $\mathcal{F}_{0}$-measurable, scalar stochastic process defined on $[-\sigma, 0)$, where $\sigma=\max \left\{\tau_{j l}, l=0, \cdots, m, j=1, \cdots, m_{l}\right\}$.

4) $b_{i}$ is an $\mathcal{F}_{0}$-measurable random variable for $i=1, \cdots, n$.

We define a solution of the initial value problem (2), (5), (6) to be a predictable stochastic process $x(t), t \geq-\sigma$, which is $(n-1)$-times differentiable on $(0, \infty)$ and which satisfies the initial conditions (5), (6) and the integral equation

$$
\begin{aligned}
x^{(n-1)}(t)= & b_{n}+\int_{o}^{t}\left[-\sum_{j=0}^{n-1} a_{j 0}(s) x^{(j)}(s)+\sum_{j=1}^{m_{0}} c_{j 0}(s) x\left(h_{j 0}(s)\right)\right] \mathrm{d} s \\
& +\sum_{l=1}^{m} \int_{o}^{t}\left[-\sum_{j=0}^{n-1} a_{j l}(s) x^{(j)}(s)+\sum_{j=1}^{m_{l}} c_{j l}(s) x\left(h_{j l}(s)\right)\right] \mathrm{d} \mathcal{B}_{l}(s)(t \geq 0),
\end{aligned}
$$

where the integrals are understood in the Lebesgue and the Itô sense, respectively, and $x\left(h_{j l}(s)\right)=\varphi\left(h_{j l}(s)\right)$ if $h_{j l}(s)<0$.

The initial value problem (2), (5), (6) has a unique (up to the natural $P$-equivalency) solution $x(t, b, \varphi)$ (see e.g. [14]). In other words, the stochastic process $x(t, b, \varphi)$ satisfies Equation (2) and the initial conditions $x(t, b, \varphi)=\varphi(t) \quad(t<0), x^{(j)}(0, b, \varphi)=b_{j+1} \quad(j=0, \cdots, n-1)$.

We will write $b:=\left(b_{1}, \cdots, b_{n}\right) \in k^{n}$, where $k^{n}$ denotes the linear space of all $n$-dimensional, $\mathcal{F}_{0}$-measurable random values. In addition, we define the following normed space:

$$
k_{p}^{n}=\left\{\alpha: \alpha \in k^{n},\|\alpha\|_{k_{p}^{n}} \equiv\left(E|\alpha|^{p}\right)^{1 / p}<\infty\right\} .
$$


Definition 1 [14] We say that Equation (2) is exponentially p-stable $(1 \leq p<\infty)$ if there are positive numbers $K, \lambda$ such that all solutions $x(t, b, \varphi)$ of the initial value problem (2), (5), (6) satisfy

$$
\left(E|x(t, b, \varphi)|^{p}\right)^{1 / p} \leq K \exp \{-\lambda t\}\left(\|b\|_{k_{p}^{n}}+\underset{t<0}{\operatorname{ess} \sup }\left(E|\varphi(t)|^{p}\right)^{1 / p}\right)(t \geq 0) .
$$

The analysis of the exponential p-stability of Equation (2) will be performed via an equivalent first order system of Itô equations. The technique of reduction of a high-order linear differential equation to a system by the substitution $x^{(k)}=x_{k+1}$ is quite common, and for system (2) it yields

$$
\begin{aligned}
& x_{j}^{\prime}(t)=x_{j+1}(t)(t \geq 0), j=1, \cdots, n-1, \\
& \mathrm{~d} x_{n}(t)=\left[-\sum_{j=0}^{n-1} a_{j 0}(t) x_{j+1}(t)+\sum_{j=1}^{m_{0}} c_{j 0}(t) x_{1}\left(h_{j 0}(t)\right)\right] \mathrm{d} t \\
& +\sum_{l=1}^{m}\left[-\sum_{j=0}^{n-1} a_{j l}(t) x_{j+1}(t)+\sum_{j=1}^{m_{l}} c_{j l}(t) x_{1}\left(h_{j l}(t)\right)\right] \mathrm{d} \mathcal{B}_{l}(t)(t \geq 0),
\end{aligned}
$$

and

$$
\begin{gathered}
x_{1}(t)=\varphi(t)(t<0), \\
x_{j}(0)=b_{j}, j=1, \cdots, n,
\end{gathered}
$$

where the first component $x_{1}$ of the solution $\left(x_{1}, \cdots, x_{n}\right)$ of initial value problem (10), (11), (12) coincides with the solution $x$ of the initial value problem (2), (5), (6), so that the exponential p-stability of Equation (2) follows from the exponential $p$-stability of system (10); and the latter can be, at least in the theory, studied by the Lyapunov-Razumikhin method of the stability analysis of stochastic delay equations. This method is based on finding a suitable Lyapunov function satisfying special conditions (see e.g. [2]), which guarantee the stability properties in question. However, practical implementation of this technique seems to be difficult.

Below we use the generalized reduction technique based on a set of positive parameters, which can be chosen arbitrarily. Adapting this set to the coefficients of the given stochastic equation considerably increases, and this will be shown in the paper, flexibility of the reduction method. In addition, we will combine this technique with the method of stability analysis based on positive invertible matrices [19] being, to our opinion, a more efficient alternative to the Lyapunov-Razumikhin algorithm, at least in the case of stochastic linear equations with delay.

Let $q_{j}(j=1, \cdots, n-1)$ be some positive numbers. Consider the following generalization of system (10):

$$
\begin{aligned}
& x_{j}^{\prime}(t)=-q_{j} x_{j}(t)+x_{j+1}(t)(t \geq 0), j=1, \cdots, n-1, \\
& \mathrm{~d} x_{n}(t)=\left[-\left(a_{(n-1) 0}(t)-\sum_{j=1}^{n-1} q_{j}\right) x_{n}(t)+\sum_{j=1}^{n-1} g_{j 0}(t) x_{j}(t)+\sum_{j=1}^{m_{0}} c_{j 0}(t) x_{1}\left(h_{j 0}(t)\right)\right] \mathrm{d} t \\
& +\sum_{l=1}^{m}\left[-a_{(n-1) l}(t) x_{n}(t)+\sum_{j=1}^{n-1} g_{j l}(t) x_{j}(t)+\sum_{j=1}^{m_{l}} c_{j l}(t) x_{1}\left(h_{j l}(t)\right)\right] \mathrm{d} \mathcal{B}_{l}(t)(t \geq 0),
\end{aligned}
$$


where $g_{j 0}(t)=\sum_{i=1}^{j} q_{i} S_{j-i, n-i, i}-\sum_{i=j-1}^{n-1} a_{i 0}(t) S_{j-1, i, 1} \quad(j=1, \cdots, n-1)$,

$$
\begin{array}{r}
g_{j l}(t)=-\sum_{i=j-1}^{n-1} a_{i l}(t) S_{j-1, i, 1} \quad(l=1, \cdots, m, j=1, \cdots, n-1) \text { and } \\
S_{r, k, i}=(-1)^{k-r} \sum_{k_{0}+\cdots+k_{r}=k-r} \prod_{j=0}^{r} q_{i+j}^{k_{j}}, S_{k, k, i}=1
\end{array}
$$

for $i+r<i+k \leq n$, and the other entries are obtained from Equation (2). System (13) is supposed to be equipped with the initial conditions (11), (12).

Let us make some comments on this reduction technique. According to the paper [17], the solution of the deterministic counterpart of the initial value problems (13), (11), (12) (i.e. in the absence of all Brownian motions) gives the solution of the (deterministic) problem (2), (5), (6) if one puts $x(t)=x_{1}(t)$. Replacing the chain rule by Itô's formula leads to the same conclusion for the stochastic initial value problem (2), (5), (6). In particular, the exponential $p$-stability of Equation (2) follows from the exponential stability of system (13) for any $1 \leq p<\infty$.

Lemma 1 Let $g(s)$ be a scalar function which is square integrable on $[0, \infty), f(s)$ be a predictable stochastic process satisfying

$$
\begin{aligned}
& \sup _{s \geq 0}\left(E|f(s)|^{2 p}\right)^{1 / 2 p}<\infty \text {. Then } \\
& \quad \sup _{s \geq 0}\left(E\left|\int_{0}^{t} g(s) f(s) \mathrm{d} s\right|^{2 p}\right)^{1 / 2 p} \leq \sup _{t \geq 0}\left(\int_{0}^{t}|g(s)| \mathrm{d} s\right) \sup _{t \geq 0}\left(E|f(t)|^{2 p}\right)^{1 / 2 p}
\end{aligned}
$$

and

$$
\sup _{t \geq 0}\left(E\left|\int_{0}^{t}(g(s))^{2}(f(s))^{2} \mathrm{~d} s\right|^{p}\right)^{1 / 2 p} \leq \sup _{t \geq 0}\left(\int_{0}^{t}(g(s))^{2} \mathrm{~d} s\right)^{1 / 2} \sup _{t \geq 0}\left(E|f(t)|^{2 p}\right)^{1 / 2 p} .
$$

Proof. Once we prove the inequality (15), the inequality (16) can be justified similarly.

$$
\begin{aligned}
& \sup _{t \geq 0}\left(E\left|\int_{0}^{t} g(s) f(s) \mathrm{d} s\right|^{2 p}\right)^{1 / 2 p} \leq \sup _{t \geq 0}\left(E\left(\int_{0}^{t}|g(s)||f(s)| \mathrm{d} s\right)^{2 p}\right)^{1 / 2 p} \\
& \leq \sup _{t \geq 0}\left(E\left(\int_{0}^{t}|g(s)|^{(2 p-1) / 2 p}|g(s)|^{1 / 2 p}|f(s)| \mathrm{d} s\right)^{2 p}\right)^{1 / 2 p} \\
& \leq \sup _{t \geq 0}\left(E\left(\left(\int_{0}^{t}|g(s)| \mathrm{d} s\right)^{2 p-1} \int_{0}^{t}|g(s)||f(s)|^{2 p} \mathrm{~d} s\right)^{1 / 2 p}\right) \\
& \leq \sup _{t \geq 0}\left(\left(\int_{0}^{t}|g(s)| \mathrm{d} s\right)^{2 p-1} \int_{0}^{t}|g(s)| E|f(s)|^{2 p} \mathrm{~d} s\right)^{1 / 2 p} \\
& \leq \sup _{t \geq 0}\left(\int_{0}^{t}|g(s)| \mathrm{d} s\right) \sup _{t \geq 0}\left(E|f(t)|^{2 p}\right)^{1 / 2 p} .
\end{aligned}
$$




\section{Main Result}

An $n \times n$-matrix $\Gamma=\left(\gamma_{i j}\right)_{i, j=1}^{n}$ is called nonnegative if $\gamma_{i j} \geq 0, i, j=1, \cdots, n$, and positive if $\gamma_{i j}>0, i, j=1, \cdots, n$.

Definition 2 A matrix $\Gamma=\left(\gamma_{i j}\right)_{i, j=1}^{n}$ is called an $\mathcal{M}$-matrix if $\gamma_{i j} \leq 0$ for $i, j=1, \cdots, n, \quad i \neq j$ and one of the following conditions is satisfied:

- $\quad \Gamma$ has a positive inverse matrix $\Gamma^{-1}$;

- the principal minors of the matrix $\Gamma$ are positive.

Now we define an $n \times n$-matrix $\Gamma$ which plays a crucial role in the theorem below. Let

- $\quad \gamma_{i i}=1, \gamma_{i(i+1)}=-\frac{1}{q_{i}}(i=1, \cdots, n-1)$,

- $\quad \gamma_{i j}=0(i=1, \cdots n-1, j=1, \cdots, n, i \neq j, j-i \neq 1)$,

$-\gamma_{n 1}=-\frac{G_{10}+\sum_{j=1}^{m_{0}} c_{j 0}}{q_{n}}-\frac{\rho_{p} \sum_{l=1}^{m}\left[G_{1 l}+\sum_{j=1}^{m_{l}} c_{j l}\right]}{\sqrt{2 q_{n}}}$,

$-\gamma_{n j}=-\frac{G_{j 0}}{q_{n}}-\frac{\rho_{p} \sum_{l=1}^{m} G_{j l}}{\sqrt{2 q_{n}}}(j=2, \cdots, n-1)$,

$-\gamma_{n n}=1-\frac{\rho_{p}\left(\sum_{l=1}^{m} A_{(n-1) l}\right)}{\sqrt{2 q_{n}}}$.

Here $\quad q_{n}=\hat{a}_{(n-1) 0}-\sum_{i=1}^{n-1} q_{j} \quad$ and $\quad G_{j l}=\sup _{t>0}\left|g_{j l}(t)\right|$ for all $j=1, \cdots, n-1$, $l=0, \cdots, m$. These numbers can be expressed via the constants $\hat{a}_{j 0}, A_{j l}$ and $q_{j}$ from assumption 1 in Section 2 . Thus, the matrix $\Gamma$ becomes

$$
\Gamma=\left(\begin{array}{cccccc}
1 & -\frac{1}{q_{1}} & 0 & \cdots & 0 & 0 \\
0 & 1 & -\frac{1}{q_{2}} & \ddots & 0 & 0 \\
0 & 0 & 1 & -\frac{1}{q_{3}} & \ddots & 0 \\
\vdots & \vdots & \vdots & \ddots & \ddots & \vdots \\
0 & 0 & 0 & \cdots & 1 & -\frac{1}{q_{n-1}} \\
\gamma_{n 1} & \gamma_{n 2} & \gamma_{n 3} & \cdots & \gamma_{n, n-1} & \gamma_{n n}
\end{array}\right) .
$$

Theorem 1 Assume that $1 \leq p<\infty$ and there exist positive numbers $q_{j},(j=1, \cdots, n-1)$ such that $q_{n}>0$ and

$$
\gamma_{n n}+\sum_{j=1}^{n-1} \gamma_{n j} \prod_{r=j}^{n-1} \frac{1}{q_{r}}>0
$$

Then system (13), and hence Equation (2), is exponentially $2 p$-stable. 
Proof. First of all, we observe that the determinant of the matrix $\Gamma$ is equal to the left-hand side of the equality (19), while the other principal minors are all equal to 1 . Hence $\Gamma$ is an $\mathcal{M}$-matrix.

Now, system (13) with the conditions (11) can be rewritten as follows:

$$
\begin{aligned}
\bar{x}_{j}(t)= & -q_{j} \bar{x}_{j}(t)+\bar{x}_{j+1}(t)(t \geq 0), j=1, \cdots, n-1, \\
\mathrm{~d} \bar{x}_{n}(t)= & {\left[-\left(a_{(n-1) 0}(t)-\sum_{j=1}^{n-1} q_{j}\right) \bar{x}_{n}(t)+\sum_{j=1}^{n-1} g_{j 0}(t) \bar{x}_{j}(t)\right.} \\
& \left.+\sum_{j=1}^{m_{0}} c_{j 0}(t)\left(\bar{x}_{1}\left(h_{j 0}(t)\right)+\bar{\varphi}\left(h_{j 0}(t)\right)\right)\right] \mathrm{d} t \\
& +\sum_{l=1}^{m}\left[-a_{(n-1) l}(t) \bar{x}_{n}(t)+\sum_{j=1}^{n-1} g_{j l}(t) \bar{x}_{j}(t)\right. \\
& \left.+\sum_{j=1}^{m_{l}} c_{j l}(t)\left(\bar{x}_{1}\left(h_{j l}(t)\right)+\bar{\varphi}\left(h_{j l}(t)\right)\right)\right] \mathrm{d} \mathcal{B}_{l}(t)(t \geq 0),
\end{aligned}
$$

where $\bar{x}_{i}(t)$ is an unknown scalar predictable stochastic process on $(-\infty, \infty)$ such that $\bar{x}_{i}(t)=0$ for $t<0$, and $\bar{\varphi}(t)$ a known scalar predictable stochastic process on $(-\infty, \infty)$ such that $\bar{\varphi}(t)=\varphi(t)$ for $t \in[-\sigma, 0)$ and $\bar{\varphi}(t)=0$ outside the interval $[-\sigma, 0)$.

Let $\bar{x}(t)=\left(\bar{x}_{1}(t), \cdots, \bar{x}_{n}(t)\right)$ be the solution of (20) satisfying the initial conditions (12). A straightforward calculation shows that $\bar{x}(t)$ coincides with the solution of the initial value problem (11), (12), (13) for $t \geq 0$ (but not necessarily for $t<0$, of course).

We choose a positive number $\lambda<\min \left\{q_{1}, \cdots, q_{n}\right\}$ for all $i=1, \cdots, n$ and make the following substitution into system $(20): \bar{x}(t)=\exp \{-\lambda t\} y(t)$, where $y(t)=\left(y_{1}(t), \cdots, y_{n}(t)\right)$ is an unknown predictable stochastic process defined on $(-\infty, \infty)$. By the definition, $y_{i}(t)=0$ for $t<0$ and $1 \leq i \leq n$, thus

$$
\begin{aligned}
& y_{j}^{\prime}(t)=\left(\lambda-q_{j}\right) y_{j}(t)+y_{j+1}(t)(t \geq 0), j=1, \cdots, n-1, \\
& \mathrm{~d} y_{n}(t)=\left[\left(\lambda-\left(a_{(n-1) 0}(t)-\sum_{j=1}^{n-1} q_{j}\right)\right) y_{n}(t)+\sum_{j=1}^{n-1} g_{j 0}(t) y_{j}(t)\right. \\
& \left.+\sum_{j=1}^{m_{0}} c_{j 0}(t) \exp \{\lambda t\}\left(\exp \left\{-\lambda h_{j 0}(t)\right\} y_{1}\left(h_{j 0}(t)\right)+\bar{\varphi}\left(h_{j 0}(t)\right)\right)\right] \mathrm{d} t \\
& +\sum_{l=1}^{m}\left[-a_{(n-1) l}(t) y_{n}(t)+\sum_{j=1}^{n-1} g_{j l}(t) y_{j}(t)\right. \\
& \left.+\sum_{j=1}^{m_{l}} c_{j l}(t) \exp \{\lambda t\}\left(\exp \left\{-\lambda h_{j l}(t)\right\} y_{1}\left(h_{j l}(t)\right)+\bar{\varphi}\left(h_{j l}(t)\right)\right)\right] \mathrm{d} \mathcal{B}_{l}(t)(t \geq 0) \text {. } \\
& \text { Let } \eta(t)=\left(a_{(n-1) 0}(t)-\sum_{j=1}^{n-1} q_{j}\right)-\lambda \text {. Then by using (12), we rewrite system (21) } \\
& y_{j}(t)=\exp \left\{-\left(q_{j}-\lambda\right) t\right\} b_{i}+\int_{0}^{t} \exp \left\{-\left(q_{j}-\lambda\right)(t-s)\right\} y_{j+1}(s) \mathrm{d} s(t \geq 0), \\
& j=1, \cdots, n-1 \text {, }
\end{aligned}
$$




$$
\begin{aligned}
& y_{n}(t)=\exp \left\{-\int_{0}^{t} \mu(s) \mathrm{d} s\right\} b_{n}+\int_{0}^{t} \exp \left\{-\int_{s}^{t} \mu(\zeta) \mathrm{d} \zeta\right\}\left[\sum_{j=1}^{n-1} g_{j 0}(s) y_{j}(s)\right. \\
& \left.+\sum_{j=1}^{m_{0}} c_{j 0}(s) \exp \{\lambda s\}\left(\exp \left\{-\lambda h_{j 0}(s)\right\} y_{1}\left(h_{j 0}(s)\right)+\bar{\varphi}\left(h_{j 0}(s)\right)\right)\right] \mathrm{d} s \\
& +\int_{0}^{t} \exp \left\{-\int_{s}^{t} \mu(\zeta) \mathrm{d} \zeta\right\} \sum_{l=1}^{m}\left[-a_{(n-1) l}(t) y_{n}(s)+\sum_{j=1}^{n-1} g_{j l}(s) y_{j}(s)\right. \\
& \left.+\sum_{j=1}^{m_{l}} c_{j l}(s) \exp \{\lambda s\}\left(\exp \left\{-\lambda h_{j l}(s)\right\} y_{1}\left(h_{j l}(s)\right)+\bar{\varphi}\left(h_{j l}(s)\right)\right)\right] \mathrm{d} \mathcal{B}_{l}(s)(t \geq 0) .
\end{aligned}
$$

Denote $\hat{y}_{i}=\sup _{t \geq 0}\left(E\left|y_{i}(t)\right|^{2 p}\right)^{1 / 2 p}$, and $\hat{\varphi}=\operatorname{ess} \sup _{t \geq 0}\left(E|\varphi(t)|^{2 p}\right)^{1 / 2 p}$

From the first $(n-1)$ equations in (22) we obtain

$$
\hat{y}_{i} \leq\left\|b_{i}\right\|_{k_{2}^{1} p}+\frac{1}{q_{i}-\lambda} \hat{y}_{i+1}, i=1, \cdots, n-1 .
$$

The estimate (4) and the last equation in (22) yield

$$
\begin{aligned}
\hat{y}_{n} \leq & \left\|b_{n}\right\|_{k_{2 p}^{1}}+\left[\sum_{j=1}^{n-1} G_{j 0} \hat{y}_{j}+\sum_{j=1}^{m_{0}} c_{j 0} \exp \left\{\lambda \tau_{j 0}\right\}\left(\hat{y}_{1}+\hat{\varphi}\right)\right] \sup _{t \geq 0}^{t} \int_{0}^{t} \exp \left\{-\int_{s}^{t} \mu(\zeta) \mathrm{d} \zeta\right\} \mathrm{d} s \\
& +\rho_{p} \sum_{l=1}^{m}\left[A_{(n-1) l} \hat{y}_{n}+\sum_{j=1}^{n-1} G_{j l}(s) \hat{y}_{j}(s)+\sum_{j=1}^{m_{l}} c_{j l} \exp \left\{\lambda \tau_{j l}\right\}\left(\hat{y}_{1}+\hat{\varphi}\right)\right] \\
& \times \sup _{t \geq 0}\left(\int_{0}^{t} \exp \left\{-2 \int_{s}^{t} \mu(\zeta) \mathrm{d} \zeta\right\} \mathrm{d} s\right)^{1 / 2} .
\end{aligned}
$$

Since

$$
\begin{aligned}
& \sup _{t \geq 0}^{t} \int_{0}^{t} \exp \left\{-\int_{s}^{t} \mu(\zeta) \mathrm{d} \zeta\right\} \mathrm{d} s \\
& =\sup _{t \geq 0} \int_{0}^{t}\left[\exp \left\{-\int_{s}^{t} \mu(\zeta) \mathrm{d} \zeta\right\} \mu(s)\right] / \mu(s) \mathrm{d} s \\
& \leq \frac{1}{\hat{a}_{(n-1) 0}-\sum_{j=1}^{n-1} q_{j}-\lambda}
\end{aligned}
$$

and

$$
\begin{aligned}
& \sup _{t \geq 0}\left(\int_{0}^{t} \exp \left\{-2 \int_{s}^{t} \mu(\zeta) \mathrm{d} \zeta\right\} \mathrm{d} s\right)^{1 / 2} \\
& =\sup _{t \geq 0}\left(\int_{0}^{t}\left[\exp \left\{-2 \int_{s}^{t} \mu(\zeta) \mathrm{d} \zeta\right\} 2 \mu(s)\right] /(2 \mu(s)) \mathrm{d} s\right)^{1 / 2} \\
& \leq \frac{1}{\sqrt{2\left(\hat{a}_{(n-1) 0}-\sum_{j=1}^{n-1} q_{j}-\lambda\right)}}
\end{aligned}
$$

the inequality (24) yields 


$$
\begin{gathered}
\hat{y}_{n} \leq\left\|b_{n}\right\|_{k_{2 p}^{1}}+\frac{\sum_{j=1}^{n-1} G_{j 0} \hat{y}_{j}+\sum_{j=1}^{m_{0}} c_{j 0} \exp \left\{\lambda \tau_{j 0}\right\} \hat{y}_{1}}{\hat{a}_{(n-1) 0}-\sum_{j=1}^{n-1} q_{j}-\lambda} \\
+\frac{\rho_{p} \sum_{l=1}^{m}\left[A_{(n-1) l} \hat{y}_{n}+\sum_{j=1}^{n-1} G_{j l}(s) \hat{y}_{j}(s)+\sum_{j=1}^{m_{l}} c_{j l} \exp \left\{\lambda \tau_{j l}\right\} \hat{y}_{1}\right]}{\sqrt{2\left(\hat{a}_{(n-1) 0}-\sum_{j=1}^{n-1} q_{j}-\lambda\right)}}+M(\lambda) \hat{\varphi},
\end{gathered}
$$

where

$$
M(\lambda):=\frac{\sum_{j=1}^{m_{0}} c_{j 0} \exp \left\{\lambda \tau_{j 0}\right\}}{\hat{a}_{(n-1) 0}-\sum_{j=1}^{n-1} q_{j}-\lambda}+\frac{\rho_{p} \sum_{l=1}^{m} \sum_{j=1}^{m_{l}} c_{j l} \exp \left\{\lambda \tau_{j l}\right\}}{\sqrt{2\left(\hat{a}_{(n-1) 0}-\sum_{j=1}^{n-1} q_{j}-\lambda\right)}} .
$$

Denote $\hat{y}=\left(\hat{y}_{1}, \cdots, \hat{y}_{n}\right)$ and define the $n \times n$--matrix $\Gamma(\lambda)=\left(\gamma_{i j}(\lambda)\right)_{i, j=1}^{n}$ by putting

- $\quad \gamma_{i i}(\lambda)=1, \gamma_{i(i+1)}(\lambda)=\frac{1}{\lambda-q_{i}}(i=1, \cdots, n-1)$,

- $\quad \gamma_{i j}(\lambda)=0(i=1, \cdots, n-1, j=1, \cdots n, i \neq j, j-i \neq 1)$,

$-\gamma_{n 1}(\lambda)=-\frac{G_{10} \exp \left\{\lambda \tau_{j 0}\right\}+\sum_{j=1}^{m_{0}} c_{j 0}}{q_{n}-\lambda}-\frac{\rho_{p} \sum_{l=1}^{m}\left[G_{1 l}+\sum_{j=1}^{m_{l}} c_{j l} \exp \left\{\lambda \tau_{j l}\right\}\right]}{\sqrt{2 q_{n}-\lambda}}$,

- $\gamma_{n j}(\lambda)=-\frac{G_{j 0}}{q_{n}-\lambda}-\frac{\rho_{p} \sum_{l=1}^{m} G_{j l}}{\sqrt{2 q_{n}-\lambda}}(j=2, \cdots, n-1)$,

- $\gamma_{n n}(\lambda)=1-\frac{\rho_{p} \sum_{l=1}^{m} A_{(n-1) l}}{\sqrt{2 q_{n}-\lambda}}$.

Then from (27) we obtain the componentwise vector inequality

$$
\Gamma(\lambda) \hat{y} \leq\left(\left\|b_{n}\right\|_{k_{2 p}^{n}}+M(\lambda) \hat{\varphi}\right) v,
$$

where $v=(1, \cdots, 1)^{\mathrm{T}}$ is an $n$-dimentional column vector.

Since $\Gamma(0)=\Gamma$ is an $\mathcal{M}$-matrix, $\Gamma(\lambda)$ is also an $\mathcal{M}$-matrix for small $\lambda$. Therefore there exists a number $\lambda=\lambda_{0}$ such that the matrix $\Gamma\left(\lambda_{0}\right)$ is positive invertible. The inequality (29) justifies

$$
|\hat{y}| \leq K\left(\left\|b_{n}\right\|_{k_{2 p}^{n}}+\hat{\varphi}\right)
$$

where $K=\left\|\Gamma\left(\lambda_{0}\right)^{-1} v\right\| \max \left\{1,\left|M\left(\lambda_{0}\right)\right|\right\}$.

Recall

$$
\bar{x}(t)=\exp \{-\lambda t\} y(t), \sup _{t \geq 0}\left(E|y(t)|^{2 p}\right)^{1 / 2 p} \leq|\hat{y}|, \hat{\varphi}=\operatorname{ess} \sup _{t \geq 0}\left(E|\varphi(t)|^{2 p}\right)^{1 / 2 p} .
$$


Based on the inequality (30), we conclude that the solution $\bar{x}(t)$ of the initial value problem (13), (11), (12) satisfies

$$
\left(E|\bar{X}(t)|^{2 p}\right)^{1 / 2 p} \leq K \exp \{-\lambda t\}\left(\|b\|_{k_{2 p}^{n}}+\operatorname{ess} \sup _{t \geq 0}\left(E|\varphi(t)|^{2 p}\right)^{1 / 2 p}\right)(t \geq 0),
$$

where $\lambda=\lambda_{0}, \quad K=\left\|\Gamma\left(\lambda_{0}\right)^{-1} v\right\| \max \left\{1,\left|M\left(\lambda_{0}\right)\right|\right\}$. Therefore system (13) is exponentially $2 p$-stable. Theorem 1 is proven.

\section{Some Corollaries}

In this section we consider a second order equation (a particular case of Equation (10) if $n=2$ )

$$
\begin{aligned}
\mathrm{d} x^{\prime}(t)= & {\left[-a_{10}(t) x^{\prime}(t)+\sum_{j=1}^{m_{0}} c_{j 0}(t) x\left(h_{j 0}(t)\right)\right] \mathrm{d} t } \\
& +\sum_{l=1}^{m}\left[-a_{0 l}(t) x^{\prime}(t)+\sum_{j=1}^{m_{l}} c_{j l}(t) x\left(h_{j l}(t)\right)\right] \mathrm{d} \mathcal{B}_{l}(t)(t \geq 0),
\end{aligned}
$$

that we transform into

$$
\begin{aligned}
x_{1}^{\prime}(t)= & -q_{1} x_{1}(t)+x_{2}(t)(t \geq 0), \\
\mathrm{d} x_{2}(t)= & {\left[-\left(a_{10}(t)-q_{1}\right) x_{2}(t)+g_{10}(t) x_{1}(t)+\sum_{j=1}^{m_{0}} c_{j 0}(t) x_{1}\left(h_{j 0}(t)\right)\right] \mathrm{d} t } \\
& +\sum_{l=1}^{m}\left[-a_{1 l}(t) x_{2}(t)+g_{1 l}(t) x_{1}(t)+\sum_{j=1}^{m_{l}} c_{j l}(t) x_{1}\left(h_{j l}(t)\right)\right] \mathrm{d} \mathcal{B}_{l}(t)(t \geq 0),
\end{aligned}
$$

where $q_{1}$ is some positive number and $g_{10}(t)=q_{1}\left(q_{1}-a_{10}(t)\right)$, $g_{1 l}(t)=-q_{1} a_{1 l}(t)-a_{0 l}(t)$ for $l=1, \cdots, m$.

The matrix $\Gamma$ is now defined as

$$
\Gamma=\left(\begin{array}{cc}
1 & -\frac{1}{q_{1}} \\
\gamma_{21} & \gamma_{22}
\end{array}\right),
$$

where

- $\gamma_{11}=1, \gamma_{12}=-\frac{1}{q_{1}}$,

$-\gamma_{21}=-\frac{G_{10}+\sum_{j=1}^{m_{0}} c_{j 0}}{q_{n}}-\frac{\rho_{p} \sum_{l=1}^{m}\left[G_{1 l}(s)+\sum_{j=1}^{m_{l}} c_{j l}\right]}{\sqrt{2 q_{2}}}$,

- $\gamma_{22}=1-\frac{\rho_{p} \sum_{l=1}^{m} A_{1 l}}{\sqrt{2 q_{2}}}$

and $a_{10}(t) \geq \bar{a}_{10}>0, \quad q_{2}=\bar{a}_{10}-q_{1}, \quad G_{1 l}=\sup \left|g_{1 l}(t)\right|$ for all $l=0, \cdots, m$.

Corollary 1 Assume that $1 \leq p<\infty$ and there exists a positive number $q_{1}<\bar{a}_{10}$ such that $q_{1} \gamma_{22}-\gamma_{21}>0$. Then system (33) is exponentially $2 p$-stable.

Proof. The statement follows from Theorem 1 and the observation that the 
determinant of the matrix $\Gamma$ is equal to $\gamma_{22}-q_{1}^{-1} \gamma_{21}>0$.

To demonstrate the efficiency of Corollary 1 , let us consider the following particular case of Equation (33):

$$
\mathrm{d} x^{\prime}(t)=\left[-a x^{\prime}(t)-b x(t)+c x(t-\tau)\right] \mathrm{d} t+\left[-\delta x^{\prime}(t)-e x(t)\right] \mathrm{d} \mathcal{B}(t)(t \geq 0),
$$

where $\mathcal{B}$ is the standard scalar Brownian motion, $a, b, c, \delta, e, \tau$ are positive real numbers. Then a straightforward application of Corollory 1 yields.

Corollary 2 Assume that $1 \leq p<\infty$ and there exists a positive number $q_{1}<a$ such that

$$
1-\frac{\rho_{p}(\delta+e)}{\sqrt{2\left(a-q_{1}\right)}}-\frac{1}{q_{1}}\left[\frac{q_{1}\left(a-q_{1}\right)+b+c}{a-q_{1}}+\frac{\rho_{p}(\delta+e)}{\sqrt{2\left(a-q_{1}\right)}}\right]>0 .
$$

Then Equation (36) is exponentially2p-stable.

\section{Conclusions and Outlook}

We studied Lyapunov stability of high-order linear stochastic Itô equations with delay using a non-Lyapunov approach, which combines the method described in the review paper [14] with the reduction technique based on the so-called $\mathcal{M}$-matrices (see e.g. [17]). This gave us an opportunity to find several efficient stability conditions in terms of the coefficients of the equations.

Solution of the following problems will complement the results of the present paper:

-Find explicit stability conditions for the 3rd and higher order stochastic delay equations in terms of their coefficients.

-Find sufficient conditions for the stability of the linear hybrid SDE with delay

$$
\begin{aligned}
\mathrm{d} x^{(n-1)}(t)= & {\left[-\sum_{j=0}^{n-1} a_{j 0}(r(t)) x^{(j)}(t)+\sum_{j=1}^{m_{0}} c_{j 0}(r(t)) x\left(h_{j 0}(t)\right)\right] \mathrm{d} t } \\
& +\sum_{l=1}^{m}\left[-\sum_{j=0}^{n-1} a_{j l}(r(t)) x^{(j)}(t)+\sum_{j=1}^{m_{l}} c_{j l}(r(t)) x\left(h_{j l}(t)\right)\right] \mathrm{d} \mathcal{B}_{l}(t)(t \geq 0),
\end{aligned}
$$

where $r(t)$ is a Markov chain with its state space $S$, which is independent of the Brownian motions $B_{l}(t)$ and which represents random switchings between different delay equations.

-Generalize the suggested framework to the case of high-order SDEs driven by an arbitrary semimartingale, rather than by the Brownian motion.

-For the van der Pol-type SDE delayed equation under perturbations of white noise

$$
\mathrm{d} x^{\prime}(t)+a x^{2 n}(t) \mathrm{d} x(t)+b \mathrm{~d} x(t-\tau)=c x^{n+1}(t) \mathrm{d} \mathcal{B}(t)(n \in N),
$$

examine stability by using the linearization criteria introduced in [23] [24] in combination with the tests obtained in this paper.

\section{Acknowledgements}

The work of the first and the third author was partially supported by the grant DDG-2015-00046 of NSERC. The work of the second and the third author was 
partially supported by the grant $\backslash \# 239070$ of the Norwegian Research Council.

\section{References}

[1] Pavliotis, G.A. (2014) Stochastic Processes and Applications. Diffusion Processes, the Fokker-Planck and Langevin Equations. Texts in Applied Mathematics, Vol. 60. Springer, Berlin.

[2] Mao, X. (2007) Stochastic Differential Equations and Applications. 2nd Edition. Horwood, Chichester.

[3] Xie, X. and Liu, L. (2012) Further Results on Output Feedback Stabilization for Stochastic High-Order Nonlinear Systems with Time-Varying Delay. Automatica, 48, 2577-2586. https://doi.org/10.1016/j.automatica.2012.06.061

[4] Zhao, L. and Xi, F. (2008) Explicit Conditions for Asymptotic Stability of Stochastic Liénard-Type Equations with Markovian Switching. Journal of Mathematical Analysis and Applications, 348, 267-273. https://doi.org/10.1016/j.jmaa.2008.07.030

[5] Zhu, F., Han, Z. and Zhang, J. (2012) Stability Analysis of Stochastic Differential Equations with Markovian Switching. Systems \& Control Letters, 61, 1209-1214. https://doi.org/10.1016/j.sysconle.2012.08.013

[6] Abou-El-Ela, A., Sadek, A. and Mahmoud, A. (2015) On the Stability of Solutions for Certain Second-Order Stochastic Delay Differential Equations. Journal Differential Equations and Control Processes, 2, 1-13.

[7] Kim, P., Jung, J., Lee, S. and Seok, J. (2013) Stability and Bifurcation Analyses of Chatter Vibrations in a Nonlinear Cylindrical Traverse Grinding Process. Journal of Sound and Vibration, 332, 3879-3896. https://doi.org/10.1016/j.jsv.2013.02.009

[8] Psillakis, H. and Alexandridis, A. (2006) Inverted Pendulum Angle Tracking Control Subject to Uncertainties and Stochastic Perturbations. IFAC Proceedings, 39, 456-461. https://doi.org/10.3182/20060906-3-IT-2910.00077

[9] Torrubia, P., Billingham, J. and Axinte, D. (2016) Stochastic Simplified Modelling of Abrasive Waterjet Footprints. Proceedings of Mathematical, Physical, and Engineering Sciences, 472, 21-86. https://doi.org/10.1098/rspa.2015.0836

[10] Ma, Q., Jiao, T. and Cui, G. (2014) Output-Feedback Control for a Class of Stochastic High-Order Feed forward Nonlinear Systems with Delay. Mathematical Problems in Engineering, Article ID: 497231.

[11] Munmuangsaen, B. and Srisuchinwong, B. (2011) Elementary Chaotic Snap Flows. Chaos, Solitons \& Fractals, 44, 995-1003.

https://doi.org/10.1016/j.chaos.2011.08.008

[12] Feng, L. and Cai, Q. (2013) Stabilization of Functional System with Markovian Switching. Applied Mathematics, 4, 37-43. https://doi.org/10.4236/am.2013.411A1006

[13] Ivanov, A., Kazmerchuk, Y. and Swishchuk, A. (2003) Theory, Stochastic Stability and Applications of Stochastic Delay Differential Equations: A Survey of Results. Differential Equations and Dynamical Systems, 11, 55-115.

[14] Kadiev, R. and Ponosov, A. (2016) Input-to-State Stability of Linear Stochastic Functional Differential Equations. Journal of Functional Spaces, 2016, Article ID: 8901563.

[15] Li, F. and Liu, Y. (2014) Global Stability and Stabilization of More General Stochastic Nonlinear Systems. Journal of Mathematical Analysis and Applications, 413, 841-855. https://doi.org/10.1016/j.jmaa.2013.12.021

[16] Yuan, C. and Mao, X. (2004) Robust Stability and Controllability of Stochastic Dif- 
ferential Delay Equations with Markovian Switching. Automatica, 40, 343-354. https://doi.org/10.1016/j.automatica.2003.10.012

[17] Amster, P. and Idels, L. (2016) New Applications of M-Matrix to Stability of High-Order Linear Delayed Equations. Applied Mathematical Letters, 54, 1-6. https://doi.org/10.1016/j.aml.2015.10.008

[18] Liao, X. (1993) Absolute Stability of Nonlinear Control Systems. In: Hazewinkel, M., Ed., Mathematics and Its Applications (Chinese Series), Kluwer Academic Publishers, Dordrecht, 1-178. https://doi.org/10.1007/978-94-017-0608-7

[19] Berezansky, L., Braverman, E. and Idels, L. (2014) New Global Exponential Stability Criteria for Nonlinear Delay Differential Systems with Applications to Bam Neural Networks. Applied Mathematics and Computation, 243, 899-910. https://doi.org/10.1016/j.amc.2014.06.060

[20] Kadiev, R. (1999) On the Stability of Stochastic Functional-Differential Equations with Respect to the First Approximation. Russian Mathematics, 43, 1-6.

[21] Ferger, D. (2014) Optimal Constants in the Marcinkiewicz-Zygmund Inequalities. Statistics \& Probability Letters, 84, 96-101. https://doi.org/10.1016/j.spl.2013.09.029

[22] Ren, Y. and Liang, H. (2001) On the Best Constant in Marcinkiewicz-Zygmund Inequality. Statistics \& Probability Letters, 53, 227-233. https://doi.org/10.1016/S0167-7152(01)00015-3

[23] Meleshko, S. and Schulz, E. (2011) Linearization of a Second-Order Stochastic Ordinary Differential Equation. Journal of Nonlinear Mathematical Physics, 18, 427-441. https://doi.org/10.1142/S1402925111001696

[24] Mkhize, T.G., Govinder, K., Moyo, S. and Meleshko, S. (2017) Linearization Criteria for Systems of Two Second-Order Stochastic Ordinary Differential Equations. Applied Mathematics and Computation, 301, 25-35. https://doi.org/10.1016/j.amc.2016.12.019 